Pacific

Journal of

Mathematics

ON THE CLASSIFICATION OF STABLE SOLUTIONS TO BIHARMONIC PROBLEMS IN LARGE DIMENSIONS

Juncheng Wei, Xingwang Xu AND Wen Yang

Volume $263 \quad$ No. 2

June 2013 


\title{
ON THE CLASSIFICATION OF STABLE SOLUTIONS TO BIHARMONIC PROBLEMS IN LARGE DIMENSIONS
}

\author{
Juncheng Wei, Xingwang Xu And Wen Yang
}

We give a new bound on the exponent for nonexistence of stable solutions to the biharmonic problem $\Delta^{2} u=u^{p}$ in $\mathbb{R}^{n}$, where $u>0, p>1$, and $n \geq 20$.

\section{Introduction}

Of concern is the biharmonic equation

$$
\Delta^{2} u=u^{p}, \quad u>0 \quad \text { in } \mathbb{R}^{n}
$$

where $n \geq 5$ and $p>1$. Set

$$
\Lambda_{u}(\varphi):=\int_{\mathbb{R}^{n}}|\Delta \varphi|^{2} d x-p \int_{\mathbb{R}^{n}} u^{p-1} \varphi^{2} d x \quad \text { for all } \varphi \in H^{2}\left(\mathbb{R}^{n}\right) .
$$

The Morse index ind $(u)$ of a classical solution to (1-1) is defined as the maximal dimension of all subspaces of $H^{2}\left(\mathbb{R}^{n}\right)$ such that $\Lambda_{u}(\varphi)<0$ in $H^{2}\left(\mathbb{R}^{n}\right) \backslash\{0\}$. We say $u$ is a stable solution to (1-1) if $\Lambda_{u}(\varphi) \geq 0$ for any test function $\varphi \in H^{2}\left(\mathbb{R}^{n}\right)$; that is, if the Morse index is zero.

In the first part of the paper, we obtain the following classification result on stable solutions of (1-1).

Theorem 1.1. Let $n \geq 20$ and $1<p<1+\frac{8 p^{*}}{n-4}$. Then (1-1) has no stable solutions.

Here $p^{*}$ stands for the smallest real root greater than $\frac{n-4}{n-8}$ of the algebraic equation

$$
\begin{aligned}
& 512(2-n) x^{6}+4\left(n^{3}-60 n^{2}+670 n-1344\right) x^{5}-2\left(13 n^{3}-424 n^{2}+3064 n-5408\right) x^{4} \\
& +2\left(27 n^{3}-572 n^{2}+3264 n-5440\right) x^{3}-\left(49 n^{3}-772 n^{2}+3776 n-5888\right) x^{2} \\
& +4\left(5 n^{3}-66 n^{2}+288 n-416\right) x-3\left(n^{3}-12 n^{2}+48 n-64\right)=0 .
\end{aligned}
$$

The first author was supported from an earmarked grant ("On Elliptic Equations with Negative Exponents") from RGC of Hong Kong.

MSC2010: primary 35B20; secondary 35J60.

Keywords: stable solutions, biharmonic superlinear equations. 
Some remarks are in order. Let us recall that for the second-order problem

$$
\Delta u+u^{p}=0 \quad u>0 \text { in } \mathbb{R}^{n}, p>1,
$$

Farina gave a complete classification of all finite Morse index solutions. The main result of [Farina 2007] is that no stable solution exists to (1-3) if either $n \leq 10, p>1$ or $n \geq 11, p<p_{J L}$. Here $p_{J L}$ denotes the Joseph-Lundgren exponent [Gui et al. 1992]. On the other hand, a stable radial solution exists for $p \geq p_{J L}$.

For the fourth-order case, the nonexistence of positive solutions to (1-1) is shown if $p<\frac{n+4}{n-4}$, and all entire solutions are classified if $p=\frac{n+4}{n-4}$. See [Lin 1998; Wei and $\mathrm{Xu}$ 1999]. When $p>\frac{n+4}{n-4}$, radially symmetric solutions to (1-1) are completely classified in [Ferrero et al. 2009; Gazzola and Grunau 2006; Guo and Wei 2010]. The radial solutions are shown to be stable if and only if $p \geq p_{J L}^{\prime}$ and $n \geq 13$, where $p_{J L}^{\prime}$ stands for the corresponding Joseph-Lundgren exponent (see [Ferrero et al. 2009; Gazzola and Grunau 2006]). In the general nonradial case, Wei and Ye [Wei and Ye 2010] showed the nonexistence of stable or finite Morse index solutions when either $n \leq 8, p>1$ or $n \geq 9, p \leq \frac{n}{n-8}$. In dimensions $n \geq 9$, a perturbation argument is used to show the nonexistence of stable solutions for $p<\frac{n}{n-8}+\varepsilon_{n}$ for some $\varepsilon_{n}>0$. However, no explicit value of $\varepsilon_{n}$ was given. The proof of Wei and Ye [2010] follows an earlier idea of Cowan, Esposito and Ghoussoub [2010] in which a similar problem in a bounded domain was studied. Theorem 1.1 gives an explicit value on $\varepsilon_{n}$ for $n \geq 20$.

In the second-order case, the proof of Farina uses basically the Moser iterations: namely multiply (1-3) by the power of $u$, like $u^{q}, q>1$. Moser iteration works because of the following simple identity

$$
\int_{\mathbb{R}^{n}} u^{q}(-\Delta u)=\frac{4 q}{(q+1)^{2}} \int_{\mathbb{R}^{n}}\left|\nabla u^{\frac{q+1}{2}}\right|^{2}, \forall u \in C_{0}^{1}\left(\mathbb{R}^{n}\right) .
$$

In the fourth-order case, such equality does not hold, and in fact we have

$$
\int_{\mathbb{R}^{n}} u^{q}\left(\Delta^{2} u\right)=\frac{4 q}{(q+1)^{2}} \int_{\mathbb{R}^{n}}\left|\Delta u^{\frac{q+1}{2}}\right|^{2}-q(q-1)^{2} \int_{\mathbb{R}^{n}} u^{q-3}|\nabla u|^{4}, \forall u \in C_{0}^{2}\left(\mathbb{R}^{n}\right) .
$$

The additional term $\int_{\mathbb{R}^{n}} u^{q-3}|\nabla u|^{4}$ makes the Moser iteration argument difficult to use. Wei and Ye [2010] used instead the new test function $-\Delta u$ and showed that $\int_{\mathbb{R}^{2}}|\Delta u|^{2}$ is bounded. Thus the exponent $\frac{n}{n-8}$ is obtained. In this paper, we use the Moser iteration for the fourth-order problem and give a control on the term $\int_{\mathbb{R}^{n}} u^{q-3}|\nabla u|^{4}$ (Lemma 2.3). As a result, we obtain a better exponent $\frac{n}{n-8}+\varepsilon_{n}$ where $\varepsilon_{n}$ is explicitly given. As far as we know, this seems to be the first result for Moser iteration for a fourth-order problem. 
In the second part of this paper, we show that the same idea can be used to establish the regularity of extremal solutions to

$$
\begin{cases}\Delta^{2} u=\lambda(u+1)^{p}, \lambda>0 & \text { in } \Omega, \\ u>0 & \text { in } \Omega, \\ u=\Delta u=0 & \text { on } \partial \Omega,\end{cases}
$$

where $\Omega$ is a smooth and bounded convex domain in $\mathbb{R}^{n}$.

For problem (1-4), it is known [Berchio and Gazzola 2005] that for $p>\frac{n+4}{n-4}$ there exists a critical value $\lambda^{*}>0$ depending on $p>1$ and $\Omega$ such that

- If $\lambda \in\left(0, \lambda^{*}\right),(1-4)$ has a minimal and classical solution which is stable;

- If $\lambda=\lambda^{*}$, a unique weak solution, called the extremal solution $u^{*}$ exists for $(1-4)$;

- No weak solution of (1-4) exists whenever $\lambda>\lambda^{*}$.

The regularity of the extremal solution of problem (1-4) at $\lambda=\lambda_{*}$ has been studied in [Cowan et al. 2010; Wei and Ye 2010], where it was shown that the extremal solution is bounded provided $n \leq 8$ or $p<\frac{n}{n-8}+\varepsilon_{n}, n \geq 9\left(\varepsilon_{n}\right.$ very small). Here, we also give a explicit bound for the exponent $p$ in large dimensions and our second result is the following.

Theorem 1.2. The extremal solution $u^{*}$ of (1-4) when $\lambda=\lambda^{*}$ is bounded provided that $n \geq 20$ and $1<p<1+\frac{8 p^{*}}{n-4}$, where $p^{*}$ is defined as above.

As $n \rightarrow+\infty$, the value $\varepsilon_{n}$ is asymptotically $8 \sqrt{8 / 3} /(n-8)^{3 / 2}$ and thus the upper bound for $p$ has the expansion

$$
1+\frac{8}{n-8}+\frac{8 \sqrt{8 / 3}}{(n-8)^{3 / 2}}+O\left(\frac{1}{(n-8)^{2}}\right)
$$

On the other hand, for radial solutions, the Joseph-Lundgren exponent [Gui et al. 1992] has the following asymptotic expansion

$$
1+\frac{8}{n-8}+\frac{16}{(n-8)^{3 / 2}}+O\left(\frac{1}{(n-8)^{2}}\right)
$$

In this paper, we have only considered fourth-order problems with power-like nonlinearity. Other kinds of nonlinearity, such as exponential and negative powers, also appear in many applications; see [Cowan et al. 2010]. However, our technique here yields no improvements of results of that reference in the case of exponential and negative nonlinearities.

This paper is organized as follows. We prove Theorem 1.1 and Theorem 1.2 respectively in Section 2 and Section 3. Some technical inequalities are given in the Appendix. 


\section{Proof of Theorem 1.1}

Lemma 2.1. For any $\varphi \in C_{0}^{4}\left(\mathbb{R}^{n}\right)$ with $\varphi \geq 0$, any $\gamma>1$ and $\varepsilon>0$ an arbitrary small number, we have

$$
\begin{gathered}
\int_{\mathbb{R}^{n}}\left(\Delta\left(u^{\gamma} \varphi^{\gamma}\right)\right)^{2} \leq \int_{\mathbb{R}^{n}}\left(\left(\Delta u^{\gamma} \varphi^{\gamma}\right)^{2}+\varepsilon|\nabla u|^{4} \varphi^{2 \gamma} u^{2 \gamma-4}+C u^{2 \gamma}\left\|\nabla^{4}\left(\varphi^{2 \gamma}\right)\right\|\right), \\
\int_{\mathbb{R}^{n}}\left(\Delta\left(u^{\gamma} \varphi^{\gamma}\right)\right)^{2} \geq \int_{\mathbb{R}^{n}}\left(\left(\Delta u^{\gamma} \varphi^{\gamma}\right)^{2}-\varepsilon|\nabla u|^{4} \varphi^{2 \gamma} u^{2 \gamma-4}-C u^{2 \gamma}\left\|\nabla^{4}\left(\varphi^{2 \gamma}\right)\right\|\right), \\
\int_{\mathbb{R}^{n}}\left(\left(u^{\gamma}\right)_{i j}\right)^{2} \varphi^{2 \gamma} \leq \int_{\mathbb{R}^{n}}\left(\left(u^{\gamma} \varphi^{\gamma}\right)_{i j}\right)^{2}+\varepsilon \int_{\mathbb{R}^{n}}|\nabla u|^{4} u^{2 \gamma-4} \varphi^{2 \gamma} \\
+C \int_{\mathbb{R}^{n}} u^{2 \gamma}\left\|\nabla^{4}\left(\varphi^{2 \gamma}\right)\right\|,
\end{gathered}
$$

where $C$ is a positive number that only depends on $\gamma$ and $\varepsilon$, and $\left\|\nabla^{4}\left(\varphi^{2 \gamma}\right)\right\|$ is defined by

$$
\left\|\nabla^{4}\left(\varphi^{2 \gamma}\right)\right\|^{2}=\varphi^{-2 \gamma}\left|\nabla \varphi^{\gamma}\right|^{4}+\left|\varphi^{\gamma}\left(\Delta^{2} \varphi^{\gamma}\right)\right|+\left|\nabla^{2} \varphi^{\gamma}\right|^{2} .
$$

In the following, unless said otherwise, the constant $C$ always denotes a positive number which may change term by term but only depends on $\gamma, \varepsilon$.

Proof. Since $\varphi$ is compactly supported, we can use integration by parts without considering the boundary terms. First, by direct calculation, we get

$$
\begin{aligned}
\left(\Delta\left(u^{\gamma} \varphi^{\gamma}\right)\right)^{2}=\left[\left(\Delta u^{\gamma}\right) \varphi^{\gamma}\right]^{2} & +4 \nabla u^{\gamma} \nabla \varphi^{\gamma} \Delta \varphi^{\gamma} u^{\gamma}+4 \nabla u^{\gamma} \nabla \varphi^{\gamma} \Delta u^{\gamma} \varphi^{\gamma} \\
& +4\left(\nabla u^{\gamma} \nabla \varphi^{\gamma}\right)^{2}+2 \Delta u^{\gamma} u^{\gamma} \Delta \varphi^{\gamma} \varphi^{\gamma}+u^{2 \gamma}\left(\Delta \varphi^{\gamma}\right)^{2} .
\end{aligned}
$$

We now need to deal with the third and fifth terms on the right side of this equality, up to the integration of both sides.

For the third term, we have

$$
\begin{aligned}
\int_{\mathbb{R}^{n}} \Delta u^{\gamma} \nabla u^{\gamma} \nabla \varphi^{\gamma} \varphi^{\gamma}=- & \int_{\mathbb{R}^{n}}\left(u^{\gamma}\right)_{i}\left(u^{\gamma}\right)_{i j}\left(\varphi^{\gamma}\right)_{j} \varphi^{\gamma} \\
& -\int_{\mathbb{R}^{n}}\left(u^{\gamma}\right)_{i}\left(u^{\gamma}\right)_{j}\left(\varphi^{\gamma}\right)_{i j} \varphi^{\gamma}-\int_{\mathbb{R}^{n}}\left(u^{\gamma}\right)_{i}\left(u^{\gamma}\right)_{j}\left(\varphi^{\gamma}\right)_{j}\left(\varphi^{\gamma}\right)_{i},
\end{aligned}
$$

where $f_{i}=\partial f / \partial x_{i}$ and $f_{i j}=\partial^{2} f / \partial x_{j} \partial x_{i}$. (Here and in the sequel, we use the Einstein summation convention, so for example $\partial_{i}\left(u_{i} u_{j} \varphi_{j}\right)=\sum_{1 \leq i, j \leq n} \partial_{i}\left(u_{i} u_{j} \varphi_{j}\right)$.) The first term on the right side of the previous equation can be estimated as

$$
\begin{aligned}
2 \int_{\mathbb{R}^{n}}\left(u^{\gamma}\right)_{i}\left(u^{\gamma}\right)_{i j}\left(\varphi^{\gamma}\right)_{j} \varphi^{\gamma}=\int_{\mathbb{R}^{n}} \partial_{j}\left(\left(u^{\gamma}\right)_{i}\left(u^{\gamma}\right)_{i}\left(\varphi^{\gamma}\right)_{j} \varphi^{\gamma}\right) & -\int_{\mathbb{R}^{n}}\left(\left(u^{\gamma}\right)_{i}\right)^{2}\left(\varphi^{\gamma}\right)_{j j} \varphi^{\gamma} \\
& -\int_{\mathbb{R}^{n}}\left(\left(u^{\gamma}\right)_{i}\right)^{2}\left(\varphi^{\gamma}\right)_{j}\left(\varphi^{\gamma}\right)_{j} .
\end{aligned}
$$


Combining these two equalities, we get

$$
\begin{aligned}
2 \int_{\mathbb{R}^{n}} \Delta u^{\gamma} \nabla u^{\gamma} \nabla \varphi^{\gamma} \varphi^{\gamma}= & -\int_{\mathbb{R}^{n}} \partial_{j}\left(\left(u^{\gamma}\right)_{i}\left(u^{\gamma}\right)_{i}\left(\varphi^{\gamma}\right)_{j} \varphi^{\gamma}\right) \\
& -\int_{\mathbb{R}^{n}} 2\left(u^{\gamma}\right)_{i}\left(u^{\gamma}\right)_{j}\left(\varphi^{\gamma}\right)_{i j} \varphi^{\gamma}-\int_{\mathbb{R}^{n}} 2\left(u^{\gamma}\right)_{i}\left(u^{\gamma}\right)_{j}\left(\varphi^{\gamma}\right)_{j}\left(\varphi^{\gamma}\right)_{i} \\
& +\int_{\mathbb{R}^{n}}\left(\left(u^{\gamma}\right)_{i}\right)^{2}\left(\varphi^{\gamma}\right)_{j j} \varphi^{\gamma}+\int_{\mathbb{R}^{n}}\left(\left(u^{\gamma}\right)_{i}\right)^{2}\left(\varphi^{\gamma}\right)_{j}\left(\varphi^{\gamma}\right)_{j} .
\end{aligned}
$$

Rewriting this equality we have

$$
\begin{aligned}
4 \int_{\mathbb{R}^{n}} \Delta u^{\gamma} \nabla u^{\gamma} \nabla \varphi^{\gamma} \varphi^{\gamma} & =2 \int_{\mathbb{R}^{n}}\left|\nabla u^{\gamma}\right|^{2} \Delta \varphi^{\gamma} \varphi^{\gamma}+2 \int_{\mathbb{R}^{n}}\left|\nabla u^{\gamma}\right|^{2}\left|\nabla \varphi^{\gamma}\right|^{2} \\
& -4 \int_{\mathbb{R}^{n}}\left(u^{\gamma}\right)_{i}\left(u^{\gamma}\right)_{j}\left(\varphi^{\gamma}\right)_{i j} \varphi^{\gamma}-4 \int_{\mathbb{R}^{n}}\left\langle\nabla u^{\gamma}, \nabla \varphi^{\gamma}\right\rangle^{2} .
\end{aligned}
$$

For the fifth term on the right side of (2-4) we have

$$
\begin{aligned}
\int_{\mathbb{R}^{n}} \Delta u^{\gamma} u^{\gamma} \Delta \varphi^{\gamma} \varphi^{\gamma}=- & \int_{\mathbb{R}^{n}} u^{\gamma}\left\langle\nabla u^{\gamma}, \nabla\left(\Delta \varphi^{\gamma}\right)\right\rangle \varphi^{\gamma} \\
& -\int_{\mathbb{R}^{n}}\left\langle\nabla u^{\gamma}, \nabla \varphi^{\gamma}\right\rangle u^{\gamma} \Delta \varphi^{\gamma}-\int_{\mathbb{R}^{n}}\left|\nabla u^{\gamma}\right|^{2} \Delta \varphi^{\gamma} \varphi^{\gamma} .
\end{aligned}
$$

Combining (2-4), (2-5) and (2-6), one obtains

$$
\begin{aligned}
\int_{\mathbb{R}^{n}}\left(\Delta\left(u^{\gamma} \varphi^{\gamma}\right)\right)^{2}-\int_{\mathbb{R}^{n}}\left(\Delta u^{\gamma}\right)^{2} \varphi^{2 \gamma} & \\
=2 \int_{\mathbb{R}^{n}}\left|\nabla u^{\gamma}\right|^{2}\left|\nabla \varphi^{\gamma}\right|^{2} & -4 \int_{\mathbb{R}^{n}} \varphi^{\gamma}\left(\nabla^{2} \varphi^{\gamma}\left(\nabla u^{\gamma}, \nabla u^{\gamma}\right)\right) \\
& +\int_{\mathbb{R}^{n}} u^{2 \gamma} \varphi^{\gamma} \Delta^{2}\left(\varphi^{\gamma}\right)-2 \int_{\mathbb{R}^{n}} u^{2 \gamma}\left(\Delta \varphi^{\gamma}\right)^{2} .
\end{aligned}
$$

Now by the Young equality, for any $\varepsilon>0$, there exists a constant $C=C(\gamma, \varepsilon)$ such that

$$
\left|\nabla u^{\gamma}\right|^{2}\left|\nabla \varphi^{\gamma}\right|^{2} \leq \frac{\varepsilon}{4}\left|\nabla u^{\gamma}\right|^{4} u^{-2 \gamma} \varphi^{2 \gamma}+C\left|\nabla \varphi^{\gamma}\right|^{4} u^{2 \gamma} \varphi^{-2 \gamma}
$$

and

$$
\left|\varphi^{\gamma}\left(\nabla^{2} \varphi^{\gamma}\left(\nabla u^{\gamma}, \nabla u^{\gamma}\right)\right)\right| \leq \frac{\varepsilon}{8}\left|\nabla u^{\gamma}\right|^{4} u^{-2 \gamma} \varphi^{2 \gamma}+C u^{2 \gamma}\left|\nabla^{2} \varphi^{\gamma}\right|^{2} .
$$

Thus by (2-7), together with the two estimates above, one gets

$$
\left|\int_{\mathbb{R}^{n}}\left(\Delta\left(u^{\gamma} \varphi^{\gamma}\right)\right)^{2}-\int_{\mathbb{R}^{n}}\left(\Delta u^{\gamma}\right)^{2} \varphi^{2 \gamma}\right| \leq \varepsilon \int_{\mathbb{R}^{n}}\left|\nabla u^{\gamma}\right|^{4} u^{-2 \gamma} \varphi^{2 \gamma}+6 C \int_{\mathbb{R}^{n}} u^{2 \gamma}\left\|\nabla^{4} \varphi^{\gamma}\right\|^{2} .
$$

The estimates (2-1) and (2-2) follow from this easily. 
Next we observe that $\left|\nabla^{2} u^{\gamma}\right|^{2} \varphi^{2 \gamma}=\left[\frac{1}{2} \Delta\left|\nabla u^{\gamma}\right|^{2}-\left\langle\nabla u^{\gamma}, \nabla \Delta u^{\gamma}\right\rangle\right] \varphi^{2 \gamma}$. Thus up to the integration by parts, with the help of (2-5) and the estimates we just proved, the estimate (2-3) also follows by noticing the identity $\int_{\mathbb{R}^{n}}\left(\Delta\left(u^{\gamma} \varphi^{\gamma}\right)\right)^{2}=$ $\int_{\mathbb{R}^{n}}\left|\nabla^{2}\left(u^{\gamma} \varphi^{\gamma}\right)\right|^{2}$. The proof of Lemma 2.1 is thus completed.

Let us return to the equation

$$
\Delta^{2} u=u^{p}, \quad u>0 \text { in } \mathbb{R}^{n} .
$$

Fix $q=2 \gamma-1>0$ and $\gamma>1$. Let $\varphi \in C_{0}^{\infty}\left(\mathbb{R}^{n}\right)$. Multiplying (2-8) by $u^{q} \varphi^{2 \gamma}$ and integration by parts, we obtain

$$
\int_{\mathbb{R}^{n}} \Delta u \Delta\left(u^{q} \varphi^{2 \gamma}\right)=\int_{\mathbb{R}^{n}} u^{p+q} \varphi^{2 \gamma}
$$

For the left side of (2-9), we have:

Lemma 2.2. For any $\varphi \in C_{0}^{\infty}\left(\mathbb{R}^{n}\right)$ with $\varphi \geq 0$, for any $\varepsilon>0$ and $\gamma$ with $q$ defined above, there exists a positive constant $C$ depends on $\gamma, \varepsilon$ such that

$$
\begin{aligned}
\int_{\mathbb{R}^{n}} \frac{\gamma^{2}}{q} \Delta u \Delta\left(u^{q} \varphi^{2 \gamma}\right) \geq \int_{\mathbb{R}^{n}}\left(\Delta u^{\gamma} \varphi^{\gamma}\right)^{2}-\int_{\mathbb{R}^{n}} C u^{2 \gamma}\left\|\nabla^{4}\left(\varphi^{2 \gamma}\right)\right\| \\
\quad-\int_{\mathbb{R}^{n}}\left(\gamma^{2}(\gamma-1)^{2}+\varepsilon\right) u^{2 \gamma-4}|\nabla u|^{4} \varphi^{2 \gamma} .
\end{aligned}
$$

Proof. First, by direct computations, we obtain

$$
\begin{array}{r}
\Delta u \Delta\left(u^{2 \gamma-1} \varphi^{2 \gamma}\right)=\Delta u\left((2 \gamma-1) u^{2 \gamma-2} \Delta u \varphi^{2 \gamma}+2(2 \gamma-1) u^{2 \gamma-2} \nabla u \nabla\left(\varphi^{2 \gamma}\right)\right. \\
\left.+(2 \gamma-1)(2 \gamma-2) u^{2 \gamma-3}|\nabla u|^{2} \varphi^{2 \gamma}+u^{2 \gamma-1} \Delta \varphi^{2 \gamma}\right), \\
\left(\Delta u^{\gamma} \varphi^{\gamma}\right)^{2}=\gamma^{2} u^{2 \gamma-2}(\Delta u)^{2} \varphi^{2 \gamma}+\gamma^{2}(\gamma-1)^{2} u^{2 \gamma-4}|\nabla u|^{4} \varphi^{2 \gamma} \\
+2(\gamma-1) \gamma^{2} u^{2 \gamma-3}|\nabla u|^{2} \Delta u \varphi^{2 \gamma} .
\end{array}
$$

Combining these two identities, we get

$$
\begin{array}{r}
\frac{\gamma^{2}}{q} \Delta u \Delta\left(u^{q} \varphi^{2 \gamma}\right)=\left(\Delta u^{\gamma} \varphi^{\gamma}\right)^{2}+2 \gamma^{2} u^{2 \gamma-2} \Delta u \\
\nabla u \nabla \varphi^{2 \gamma}+\frac{\gamma^{2}}{q} u^{2 \gamma-1} \Delta u \Delta \varphi^{2 \gamma} \\
-\gamma^{2}(\gamma-1)^{2} u^{2 \gamma-4}|\nabla u|^{4} \varphi^{2 \gamma}
\end{array}
$$

For the term $u^{2 \gamma-2} \Delta u \nabla u \nabla \varphi^{2 \gamma}$, we have

$$
\begin{aligned}
u^{2 \gamma-2} \Delta u \nabla u \nabla \varphi^{2 \gamma}=\partial_{i}\left(u^{2 \gamma-2} u_{i} u_{j}\left(\varphi^{2 \gamma}\right)_{j}\right)-(2 \gamma-2) u^{2 \gamma-3}\left(u_{i}\right)^{2} u_{j}\left(\varphi^{2 \gamma}\right)_{j} \\
-u^{2 \gamma-2} u_{i} u_{i j}\left(\varphi^{2 \gamma}\right)_{j}-u^{2 \gamma-2} u_{i} u_{j}\left(\varphi^{2 \gamma}\right)_{i j} .
\end{aligned}
$$


We can regroup the term $u^{2 \gamma-2} u_{i} u_{i j}\left(\varphi^{2 \gamma}\right)_{j}$ as

$$
\begin{aligned}
2 u^{2 \gamma-2} u_{i} u_{i j}\left(\varphi^{2 \gamma}\right)_{j}=\partial_{j}\left(u^{2 \gamma-2}\left(u_{i}\right)^{2}\left(\varphi^{2 \gamma}\right)_{j}\right)-(2 \gamma-2) u^{2 \gamma-3} u_{j}\left(u_{i}\right)^{2}\left(\varphi^{2 \gamma}\right)_{j} \\
-u^{2 \gamma-2}\left(u_{i}\right)^{2}\left(\varphi^{2 \gamma}\right)_{j j} .
\end{aligned}
$$

Therefore we get

$$
\begin{aligned}
2 u^{2 \gamma-2} \Delta u \nabla u \nabla \varphi^{2 \gamma} & =2 \partial_{i}\left(u^{2 \gamma-2} u_{i} u_{j}\left(\varphi^{2 \gamma}\right)_{j}\right)-\partial_{j}\left(u^{2 \gamma-2}\left(u_{i}\right)^{2}\left(\varphi^{2 \gamma}\right)_{j}\right) \\
& -(2 \gamma-2) u^{2 \gamma-3}\left(u_{i}\right)^{2} u_{j}\left(\varphi^{2 \gamma}\right)_{j}+u^{2 \gamma-2}\left(u_{i}\right)^{2}\left(\varphi^{2 \gamma}\right)_{j j} \\
& -2 u^{2 \gamma-2} u_{i} u_{j}\left(\varphi^{2 \gamma}\right)_{i j} .
\end{aligned}
$$

For the last three terms on the right side of (2-12), applying Young's inequality, we get

$$
\begin{aligned}
\left|u^{2 \gamma-3}\left(u_{i}\right)^{2} u_{j}\left(\varphi^{2 \gamma}\right)_{j}\right| & \leq \frac{\varepsilon}{6 \gamma^{2}(\gamma-1)} u^{2 \gamma-4}|\nabla u|^{4} \varphi^{2 \gamma}+C u^{2 \gamma}\left\|\nabla^{4}\left(\varphi^{2 \gamma}\right)\right\|, \\
\left|u^{2 \gamma-2}\left(u_{i}\right)^{2}\left(\varphi^{2 \gamma}\right)_{j j}\right| & \leq \frac{\varepsilon}{6 \gamma^{2}} u^{2 \gamma-4}|\nabla u|^{4} \varphi^{2 \gamma}+C u^{2 \gamma}\left\|\nabla^{4}\left(\varphi^{2 \gamma}\right)\right\|, \\
\left|u^{2 \gamma-2} u_{i} u_{j}\left(\varphi^{2 \gamma}\right)_{i j}\right| & \leq \frac{\varepsilon}{6 \gamma^{2}} u^{2 \gamma-4}|\nabla u|^{4} \varphi^{2 \gamma}+C u^{2 \gamma}\left\|\nabla^{4}\left(\varphi^{2 \gamma}\right)\right\| .
\end{aligned}
$$

These three inequalities and (2-12) imply

$$
\int_{\mathbb{R}^{n}} 2 \gamma^{2} u^{2 \gamma-2} \Delta u \nabla u \nabla \varphi^{2 \gamma} \geq-\frac{\varepsilon}{2} \int_{\mathbb{R}^{n}} u^{2 \gamma-4}|\nabla u|^{4} \varphi^{2 \gamma}-C \int_{\mathbb{R}^{n}} u^{2 \gamma}\left\|\nabla^{4}\left(\varphi^{2 \gamma}\right)\right\| .
$$

Similarly we get

$$
\int_{\mathbb{R}^{n}} \frac{\gamma^{2}}{q} u^{2 \gamma-1} \Delta u \Delta \varphi^{2 \gamma} \geq-\frac{\varepsilon}{2} \int_{\mathbb{R}^{n}} u^{2 \gamma-4}|\nabla u|^{4} \varphi^{2 \gamma}-C \int_{\mathbb{R}^{n}} u^{2 \gamma}\left\|\nabla^{4}\left(\varphi^{2 \gamma}\right)\right\| .
$$

Inequality (2-10) follows from (2-11), (2-13) and (2-14).

As a result of (2-1) and (2-10), we have

$$
\begin{aligned}
\int_{\mathbb{R}^{n}} \frac{\gamma^{2}}{q} \Delta u \Delta\left(u^{q} \varphi^{2 \gamma}\right) \geq \int_{\mathbb{R}^{n}}\left(\Delta\left(u^{\gamma} \varphi^{\gamma}\right)\right)^{2}-\int_{\mathbb{R}^{n}} C u^{2 \gamma}\left\|\nabla^{4}\left(\varphi^{2 \gamma}\right)\right\| \\
-\int_{\mathbb{R}^{n}}\left(\gamma^{2}(\gamma-1)^{2}+\varepsilon\right) u^{2 \gamma-4}|\nabla u|^{4} \varphi^{2 \gamma}
\end{aligned}
$$

Next we estimate the most difficult term, $\int_{\mathbb{R}^{n}} u^{2 \gamma-4}|\nabla u|^{4} \varphi^{2 \gamma}$, in (2-15). This is the key step in proving Theorem 1.1.

Lemma 2.3. If $u$ is the classical solution to the biharmonic equation (2-8), and $\varphi$ is defined as above, then for any sufficiently small $\varepsilon>0$, we have the following 
inequality

$$
\begin{aligned}
\left(\frac{1}{2}-\varepsilon\right) \int_{\mathbb{R}^{n}} u^{2 \gamma-4}|\nabla u|^{4} \varphi^{2 \gamma} \leq & \frac{2}{\gamma^{2}} \int_{\mathbb{R}^{n}}\left(\Delta\left(u^{\gamma} \varphi^{\gamma}\right)\right)^{2}+\int_{\mathbb{R}^{n}} C u^{2 \gamma}\left\|\nabla^{4}\left(\varphi^{2 \gamma}\right)\right\| \\
& -\int_{\mathbb{R}^{n}} \frac{4}{(4 \gamma-3+p)(p+1)} u^{2 \gamma+p-1} \varphi^{2 \gamma}
\end{aligned}
$$

Proof. It is easy to see that

$$
\int_{\mathbb{R}^{n}} u^{2 \gamma-4}|\nabla u|^{4} \varphi^{2 \gamma}=\frac{1}{\gamma^{4}} \int_{\mathbb{R}^{n}} u^{-2 \gamma}\left|\nabla u^{\gamma}\right|^{4} \varphi^{2 \gamma},
$$

and

$$
\begin{aligned}
\int_{\mathbb{R}^{n}} u^{-2 \gamma} & \left|\nabla u^{\gamma}\right|^{4} \varphi^{2 \gamma} \\
& =\int_{\mathbb{R}^{n}} u^{-2 \gamma}\left|\nabla u^{\gamma}\right|^{2} \nabla u^{\gamma} \nabla u^{\gamma} \varphi^{2 \gamma} \\
& =\int_{\mathbb{R}^{n}}-\nabla u^{-\gamma}\left|\nabla u^{\gamma}\right|^{2} \nabla u^{\gamma} \varphi^{2 \gamma} \\
& =\int_{\mathbb{R}^{n}} u^{-\gamma}\left|\nabla u^{\gamma}\right|^{2} \Delta u^{\gamma} \varphi^{2 \gamma}+\int_{\mathbb{R}^{n}} u^{-\gamma} \nabla\left(\left|\nabla u^{\gamma}\right|^{2}\right) \nabla u^{\gamma} \varphi^{2 \gamma} \\
& +\int_{\mathbb{R}^{n}} u^{-\gamma}\left|\nabla u^{\gamma}\right|^{2} \nabla u^{\gamma} \nabla \varphi^{2 \gamma},
\end{aligned}
$$

where in the last step we used integration by parts. For the first term in the last part of this equality, we have

$$
\int_{\mathbb{R}^{n}} u^{-\gamma}\left|\nabla u^{\gamma}\right|^{2} \Delta u^{\gamma} \varphi^{2 \gamma}=\gamma^{3} \int_{\mathbb{R}^{n}}\left((\gamma-1) u^{2 \gamma-4}|\nabla u|^{4} \varphi^{2 \gamma}+u^{2 \gamma-3}|\nabla u|^{2} \Delta u \varphi^{2 \gamma}\right) .
$$

Substituting this into (2-18) and combining with (2-17), we obtain

$$
\begin{aligned}
\int_{\mathbb{R}^{n}} u^{2 \gamma-4}|\nabla u|^{4} \varphi^{2 \gamma} & =\int_{\mathbb{R}^{n}} \frac{1}{\gamma^{3}} u^{-\gamma} \nabla\left(\left|\nabla u^{\gamma}\right|^{2}\right) \nabla u^{\gamma} \varphi^{2 \gamma} \\
+ & \int_{\mathbb{R}^{n}} u^{2 \gamma-3}\left(|\nabla u|^{2}\right) \Delta u \varphi^{2 \gamma}+\int_{\mathbb{R}^{n}} \frac{1}{\gamma^{3}} u^{-\gamma}\left(\left|\nabla u^{\gamma}\right|^{2}\right) \nabla u^{\gamma} \nabla \varphi^{2 \gamma} .
\end{aligned}
$$

The first term on the right side of (2-19) can be estimated as

$$
\begin{aligned}
u^{-\gamma} \nabla\left(\left|\nabla u^{\gamma}\right|^{2}\right) \nabla u^{\gamma} & =2 u^{-\gamma}\left(\left(u^{\gamma}\right)_{i j}\left(u^{\gamma}\right)_{i}\left(u^{\gamma}\right)_{j}\right) \\
& \leq 2 \gamma\left(u^{\gamma}\right)_{i j}\left(u^{\gamma}\right)_{i j}+\frac{u^{-2 \gamma}}{2 \gamma}\left(u^{\gamma}\right)_{i}\left(u^{\gamma}\right)_{j}\left(u^{\gamma}\right)_{i}\left(u^{\gamma}\right)_{j} \\
& =2 \gamma\left|\nabla^{2} u^{\gamma}\right|^{2}+\frac{u^{-2 \gamma}}{2 \gamma}\left|\nabla u^{\gamma}\right|^{4} .
\end{aligned}
$$


As a consequence, we have

$$
\begin{aligned}
& \int_{\mathbb{R}^{n}} \frac{1}{\gamma^{3}} u^{-\gamma} \nabla\left(\left|\nabla u^{\gamma}\right|^{2}\right) \nabla u^{\gamma} \varphi^{2 \gamma} \\
& \leq \int_{\mathbb{R}^{n}} \frac{2}{\gamma^{2}}\left|\nabla^{2} u^{\gamma}\right|^{2} \varphi^{2 \gamma}+\int_{\mathbb{R}^{n}} \frac{1}{2 \gamma^{4}} u^{-2 \gamma}\left|\nabla u^{\gamma}\right|^{4} \varphi^{2 \gamma} \\
& \leq \int_{\mathbb{R}^{n}} \frac{2}{\gamma^{2}}\left|\nabla^{2}\left(u^{\gamma} \varphi^{\gamma}\right)\right|^{2}+\int_{\mathbb{R}^{n}} C u^{2 \gamma}\left\|\nabla^{4}\left(\varphi^{2 \gamma}\right)\right\|+\int_{\mathbb{R}^{n}} \frac{1+4 \gamma^{2} \varepsilon}{2 \gamma^{4}} u^{-2 \gamma}\left|\nabla u^{\gamma}\right|^{4} \varphi^{2 \gamma} \\
& =\int_{\mathbb{R}^{n}} \frac{2}{\gamma^{2}}\left(\Delta\left(u^{\gamma} \varphi^{\gamma}\right)\right)^{2}+\int_{\mathbb{R}^{n}} C u^{2 \gamma}\left\|\nabla^{4}\left(\varphi^{2 \gamma}\right)\right\|+\int_{\mathbb{R}^{n}} \frac{1+4 \gamma^{2} \varepsilon}{2 \gamma^{4}} u^{-2 \gamma}\left|\nabla u^{\gamma}\right|^{4} \varphi^{2 \gamma},
\end{aligned}
$$

where we used (2-3) in the last step.

For the second term on the right side of (2-19), applying estimate (2.3) from [Wei and Ye 2010], that is, $(\Delta u)^{2} \geq \frac{2}{p+1} u^{p+1}$, and the fact that $\Delta u<0$ from Theorem 3.1 in [Wei and Xu 1999] or Theorem 2.1 in [Xu 2000], we have

$$
\begin{aligned}
\int_{\mathbb{R}^{n}} u^{2 \gamma-3}\left(|\nabla u|^{2}\right) \Delta u \varphi^{2 \gamma} \leq & -\int_{\mathbb{R}^{n}} \sqrt{\frac{2}{p+1}} u^{2 \gamma-3+\frac{p+1}{2}}\left(|\nabla u|^{2}\right) \varphi^{2 \gamma} \\
= & \int_{\mathbb{R}^{n}} \frac{\sqrt{\frac{2}{p+1}}}{2 \gamma-2+\frac{p+1}{2}} u^{2 \gamma-2+\frac{p+1}{2}} \Delta u \varphi^{2 \gamma} \\
& +\int_{\mathbb{R}^{n}} \frac{\sqrt{\frac{2}{p+1}}}{2 \gamma-2+\frac{p+1}{2}} u^{2 \gamma-2+\frac{p+1}{2}} \nabla u \nabla \varphi^{2 \gamma} .
\end{aligned}
$$

Using the inequality $-\Delta u \geq \sqrt{\frac{2}{p+1}} u^{\frac{p+1}{2}}$, we get

$$
\int_{\mathbb{R}^{n}} \frac{\sqrt{\frac{2}{p+1}}}{2 \gamma-2+\frac{2}{p+1}} u^{2 \gamma-2+\frac{p+1}{2}} \Delta u \varphi^{2 \gamma} \leq-\int_{\mathbb{R}^{n}} \frac{\frac{2}{p+1}}{2 \gamma-2+\frac{p+1}{2}} u^{2 \gamma+p-1} \varphi^{2 \gamma} .
$$

On the other hand, for the second term on the right side of (2-22), we have

$$
\begin{aligned}
\int_{\mathbb{R}^{n}} u^{2 \gamma-2+\frac{p+1}{2}} \nabla u \nabla \varphi^{2 \gamma}= & -\int_{\mathbb{R}^{n}} \frac{1}{L} u^{2 \gamma-1+\frac{p+1}{2}} \Delta \varphi^{2 \gamma} \\
= & -\int_{\left\{x \mid \Delta \varphi^{2 \gamma}>0\right\}} \frac{1}{L} u^{2 \gamma-1+\frac{p+1}{2}} \Delta \varphi^{2 \gamma} \\
& -\int_{\left\{x \mid \Delta \varphi^{2 \gamma} \leq 0\right\}} \frac{1}{L} u^{2 \gamma-1+\frac{p+1}{2}} \Delta \varphi^{2 \gamma},
\end{aligned}
$$

where the first equality follows from integration by parts and $L=2 \gamma-1+\frac{p+1}{2}$. As for the first term on the last part of (2-24), using the inequality

$$
\Delta u \leq-\sqrt{\frac{2}{p+1}} u^{\frac{p+1}{2}}<0
$$


we have

$$
\frac{\sqrt{\frac{p+1}{2}}}{L} \int_{\left\{x \mid \Delta \varphi^{2 \gamma}>0\right\}} u^{2 \gamma-1} \Delta u \Delta \varphi^{2 \gamma} \leq-\int_{\left\{x \mid \Delta \varphi^{2 \gamma}>0\right\}} \frac{1}{L} u^{2 \gamma-1+\frac{p+1}{2}} \Delta \varphi^{2 \gamma} .
$$

Similarly to the proof of Lemma 2.1, it is easy to get

$\left|\int_{\left\{x \mid \Delta \varphi^{2 \gamma}>0\right\}} \frac{\sqrt{\frac{p+1}{2}}}{L} u^{2 \gamma-1} \Delta u \Delta \varphi^{2 \gamma}\right| \leq \varepsilon \int_{\mathbb{R}^{n}} u^{2 \gamma-4}|\nabla u|^{4} \varphi^{2 \gamma}+\int_{\mathbb{R}^{n}} C u^{2 \gamma}\left\|\nabla^{4}\left(\varphi^{2 \gamma}\right)\right\|$.

From this and (2-25), we have

$\left|\int_{\left\{x \mid \Delta \varphi^{2 \gamma}>0\right\}} \frac{1}{L} u^{2 \gamma-1+\frac{p+1}{2}} \Delta \varphi^{2 \gamma}\right| \leq \varepsilon \int_{\mathbb{R}^{n}} u^{2 \gamma-4}|\nabla u|^{4} \varphi^{2 \gamma}+\int_{\mathbb{R}^{n}} C u^{2 \gamma}\left\|\nabla^{4}\left(\varphi^{2 \gamma}\right)\right\|$.

Similarly, we also obtain

$\left|\int_{\left\{x \mid \Delta \varphi^{2 \gamma} \leq 0\right\}} \frac{1}{L} u^{2 \gamma-1+\frac{p+1}{2}} \Delta \varphi^{2 \gamma}\right| \leq \varepsilon \int_{\mathbb{R}^{n}} u^{2 \gamma-4}|\nabla u|^{4} \varphi^{2 \gamma}+\int_{\mathbb{R}^{n}} C u^{2 \gamma}\left\|\nabla^{4}\left(\varphi^{2 \gamma}\right)\right\|$.

From the last two inequalities and (2-24), we have

$$
\left|\int_{\mathbb{R}^{n}} u^{2 \gamma-2+\frac{p+1}{2}} \nabla u \nabla \varphi^{2 \gamma}\right| \leq \varepsilon \int_{\mathbb{R}^{n}} u^{2 \gamma-4}|\nabla u|^{4} \varphi^{2 \gamma}+\int_{\mathbb{R}^{n}} C u^{2 \gamma}\left\|\nabla^{4}\left(\varphi^{2 \gamma}\right)\right\| .
$$

Combining (2-22), (2-23) and (2-26), we get the inequality

$$
\begin{aligned}
\int_{\mathbb{R}^{n}} u^{2 \gamma-3}|\nabla u|^{2} \Delta u \varphi^{2 \gamma} \leq \varepsilon \int_{\mathbb{R}^{n}} u^{2 \gamma-4}|\nabla u|^{4} \varphi^{2 \gamma}+\int_{\mathbb{R}^{n}} C u^{2 \gamma}\left\|\nabla^{4}\left(\varphi^{2 \gamma}\right)\right\| \\
-\int_{\mathbb{R}^{n}} \frac{4}{(4 \gamma-3+p)(p+1)} u^{2 \gamma+p-1} \varphi^{2 \gamma} .
\end{aligned}
$$

Finally, we apply Young's inequality to the third term on the right side of (2-19), and get

$$
\begin{aligned}
\int_{\mathbb{R}^{n}} \frac{1}{\gamma^{3}} u^{-\gamma}\left(\left|\nabla u^{\gamma}\right|^{2}\right) \nabla u^{\gamma} & \nabla \varphi^{2 \gamma} \\
& =\int_{\mathbb{R}^{n}} u^{2 \gamma-3}|\nabla u|^{2} \nabla u \nabla\left(\varphi^{2 \gamma}\right) \\
& \leq \varepsilon \int_{\mathbb{R}^{n}} u^{2 \gamma-4}|\nabla u|^{4} \varphi^{2 \gamma}+\int_{\mathbb{R}^{n}} C u^{2 \gamma}\left\|\nabla^{4}\left(\varphi^{2 \gamma}\right)\right\| .
\end{aligned}
$$

By (2-19), (2-21), (2-27) and (2-28), we finally obtain

$$
\begin{aligned}
&\left(\frac{1}{2}-\varepsilon\right) \int_{\mathbb{R}^{n}} u^{2 \gamma-4}|\nabla u|^{4} \varphi^{2 \gamma} \leq \frac{2}{\gamma^{2}} \int_{\mathbb{R}^{n}}\left(\Delta\left(u^{\gamma} \varphi^{\gamma}\right)\right)^{2}+\int_{\mathbb{R}^{n}} C u^{2 \gamma}\left\|\nabla^{4}\left(\varphi^{2 \gamma}\right)\right\| \\
&-\int_{\mathbb{R}^{n}} \frac{4}{(4 \gamma-3+p)(p+1)} u^{2 \gamma+p-1} \varphi^{2 \gamma} .
\end{aligned}
$$


By (2-9), (2-15) and (2-16), since the number $\varepsilon$ is arbitrary small in those three places, we have, for $\delta>0$ sufficiently small,

$$
\begin{aligned}
& \int_{\mathbb{R}^{n}}\left(1-4(\gamma-1)^{2}-\delta\right)\left(\Delta\left(u^{\gamma} \varphi^{\gamma}\right)\right)^{2} \\
& -\int_{\mathbb{R}^{n}}\left(\frac{\gamma^{2}}{2 \gamma-1}-\frac{8 \gamma^{2}(\gamma-1)^{2}}{(4 \gamma-3+p)(p+1)}\right) u^{p+2 \gamma-1} \varphi^{2 \gamma} \leq \int_{\mathbb{R}^{n}} C_{\delta} u^{2 \gamma}\left\|\nabla^{4}\left(\varphi^{2 \gamma}\right)\right\|,
\end{aligned}
$$

where $C_{\delta}$ is a positive constant that depends on $\delta$ only. Here, we need to require $1-4(\gamma-1)^{2}>0$, since we have assumed that $\gamma>1$ in Lemma 2.1. So $\gamma$ is required be in $\left(1, \frac{3}{2}\right)$. If we can choose $\delta$ small enough to make $1-4(\gamma-1)^{2}-\delta$ positive, by the stability property of function $u$, we obtain

$$
\int_{\mathbb{R}^{n}}(E-p \delta) u^{p+q} \varphi^{2 \gamma} \leq \int_{\mathbb{R}^{n}} C_{\delta} u^{2 \gamma}\left\|\nabla^{4}\left(\varphi^{2 \gamma}\right)\right\|,
$$

where $E$ is defined to be

$$
E=p\left(1-4(\gamma-1)^{2}\right)-\frac{\gamma^{2}}{q}+\frac{8 \gamma^{2}(\gamma-1)^{2}}{(4 \gamma-3+p)(p+1)} .
$$

Now we take $\varphi=\eta^{m}$ with $m$ sufficiently large, and choose $\eta$ a cut-off function satisfying $0 \leq \eta \leq 1, \eta=1$ for $|x|<R$ and $\eta=0$ for $|x|>2 R$. By Young's inequality again, we have

$$
\begin{aligned}
\int_{\mathbb{R}^{n}} u^{2 \gamma}\left\|\nabla^{4}\left(\varphi^{2 \gamma}\right)\right\| & \leq C_{\delta} R^{-4} \int_{\mathbb{R}^{n}} u^{2 \gamma} \eta^{2 \gamma m-4} \\
& \leq C_{\delta, \varepsilon} R^{-\frac{4}{1-\theta}} \int_{\mathbb{R}^{n}} u^{2} \eta^{2 \gamma m-\frac{4}{1-\theta}}+\varepsilon C_{\delta} \int_{\mathbb{R}^{n}} u^{2 \gamma+p-1} \eta^{2 \gamma m},
\end{aligned}
$$

where $C_{\delta, \varepsilon}$ is a positive constant depends on $\delta$ and $\varepsilon$, and $\theta$ is a number such that $2(1-\theta)+(2 \gamma+p-1) \theta=2 \gamma$, so that $0<\theta<1$ for $2<2 \gamma<2 \gamma+p-1$. By (2-30) and (2-32), we get

$$
\left(E-p \delta-\varepsilon C_{\delta}\right) \int_{\mathbb{R}^{n}} u^{p+2 \gamma-1} \eta^{2 \gamma m} \leq C_{\delta, \varepsilon} R^{-\frac{4}{1-\theta}} \int_{\mathbb{R}^{n}} u^{2} \eta^{2 \gamma m-\frac{4}{1-\theta}} .
$$

Since $\theta$ is strictly less than 1 and will be fixed for given $\gamma, p$, we can choose $m$ sufficiently large to make $2 \gamma m-\frac{4}{1-\theta}>0$. On the other hand, if $E>0$, we can find small $\delta$ and then small $\varepsilon$, such that $E-p \delta-\varepsilon C_{\delta}>0$. Therefore, by the definition of function $\eta$ and (2-33), we obtain

$$
\left(E-p \delta-\varepsilon C_{\delta}\right) \int_{B_{R}} u^{p+2 \gamma-1} \leq C_{\delta, \varepsilon} R^{-\frac{4}{1-\theta}} \int_{B_{2 R}} u^{2} .
$$

By (2.10) of [Wei and Ye 2010], we have $\int_{B_{2 R}} u^{2} \leq C R^{n-\frac{8}{p-1}}$, as a result, the 
left side of (2-34) is less equal than $C_{\delta, \varepsilon} R^{n-\frac{8}{p-1}-\frac{4}{1-\theta}}$, which tends to 0 as $R$ tends to $\infty$, provided the power $n-\frac{8}{p-1}-\frac{4}{1-\theta}$ is negative, which is equivalent to $(p+2 \gamma-1)>(p-1) \frac{n}{4}$ according to the definition of $\theta$. So, if $(p+2 \gamma-1)>(p-1) \frac{n}{4}$ and $E-p \delta-C_{\delta} \varepsilon>0$, we have $u \equiv 0$.

Thus, we have proved the nonexistence of stable solution to (2-8) if $p$ satisfies the condition $(p+2 \gamma-1)>(p-1) \frac{n}{4}$ and $E>0$ (for $\delta, \varepsilon$ are arbitrary small). By Lemma A.2 in the Appendix, the power $p$ can be in the interval $\left(\frac{n}{n-8}, 1+\frac{8 p^{*}}{n-4}\right)$. Combining with Theorem 1.1 of [Wei and Ye 2010], we have proved Theorem 1.1, that is, for any $1<p<1+\frac{8 p^{*}}{n-4}, n \geq 20,(2-8)$ has no stable solution.

\section{Proof of Theorem 1.2}

In proving Theorem 1.2, it is enough to consider stable solutions $u_{\lambda}$ to (1-4), since $u^{*}=\lim _{\lambda \rightarrow \lambda^{*}} u_{\lambda}$. Now we give a uniform bound for the stable solutions to (1-4) when $0<d<\lambda<\lambda^{*}$, where $d$ is a fixed positive constant from $\left(0, \lambda^{*}\right)$.

First, we need to analyze the solution near the boundary. Specifically, we need the regularity of the stable solutions of the equation

$$
\begin{cases}\Delta^{2} u=\lambda(u+1)^{p}, \lambda>0 & \text { in } \Omega, \\ u>0 & \text { in } \Omega, \\ u=\Delta u=0 & \text { on } \partial \Omega .\end{cases}
$$

near the boundary (as well as their derivatives; see remark after the next theorem).

Theorem 3.1. Let $\Omega$ be a bounded, smooth, and convex domain. There exists a constant $C$ (independent of $\lambda, u$ ) and small positive number $\varepsilon$, such that for stable solutions $u$ to (3-1) we have

$$
u(x)<C \text { for all } x \in \Omega_{\varepsilon}:=\{z \in \Omega: d(z, \partial \Omega)<\varepsilon\} .
$$

Proof. This result is well known. See [Guo and Wei 2009]. For the sake of completeness, we include a proof here. By Lemma 3.5 of [Cowan et al. 2010], we see that there exists a constant $C$ independent of $\lambda, u$, such that

$$
\int_{\Omega}(1+u)^{p} d x \leq C
$$

We write (3-1) as

$$
\begin{cases}\Delta u+v=0, & \text { in } \Omega, \\ \Delta v+\lambda(1+u)^{p}=0, & \text { in } \Omega, \\ u=v=0, & \text { in } \partial \Omega .\end{cases}
$$

If we set $f_{1}(u, v)=v, f_{2}(u, v)=\lambda(u+1)^{p}$, we see that $\partial f_{1} / \partial v=1>0$ and $\partial f_{2} / \partial u=\lambda p(u+1)^{p-1}>0$. Therefore, the convexity of $\Omega$, Lemma 5.1 of [Troy 1981], and the moving plane method near $\partial \Omega$ (as in the appendix of [Guo and 
Webb 2002]) imply that there exist $t_{0}>0$ and $\alpha$ which depends only on the domain $\Omega$, such that $u(x-t v)$ and $v(x-t v)$ are nondecreasing for $t \in\left[0, t_{0}\right], \quad v \in \mathbb{R}^{n}$ satisfying $|\nu|=1$ and $(\nu, n(x)) \geq \alpha$ and $x \in \partial \Omega$. Therefore, we can find $\rho, \varepsilon>0$ such that for any $x \in \Omega_{\varepsilon}:=\{z \in \Omega: d(z, \partial \Omega)<\varepsilon\}$ there exists a fixed-sized cone $\Gamma_{x}$ (with $\mathrm{x}$ as its vertex) with

- $\operatorname{meas}\left(\Gamma_{x}\right) \geq \rho$,

- $\Gamma_{x} \subset\{z \in \Omega: d(z, \partial \Omega)<2 \varepsilon\}$, and

- $u(y) \geq u(x)$ for any $y \in \Gamma_{x}$.

Then, for any $x \in \Omega_{\varepsilon}$, we have

$$
(1+u(x))^{p} \leq \frac{1}{\operatorname{meas}\left(\Gamma_{x}\right)} \int_{\Gamma_{x}}(1+u)^{p} \leq \frac{1}{\rho} \int_{\Omega}(1+u)^{p} \leq C .
$$

This implies that $(1+u(x))^{p} \leq C$, therefore $u(x) \leq C$.

Remark. By classical elliptic regularity theory, $u(x)$ and its derivatives up to fourth order are bounded on the boundary by a constant independent of $u$. See [Wei 1996] for more details.

We now turn to the proof of Theorem 1.2 proper, using the ideas of Section 2. Multiplying (1-4) by $(u+1)^{q}$ and integrating by parts, we have

$$
\int_{\Omega} \lambda(u+1)^{p+q}=\int_{\Omega} \Delta^{2} u(u+1)^{q}=\int_{\partial \Omega} \frac{\partial(\Delta u)}{\partial n}+\int_{\Omega} \Delta(u+1) \Delta(u+1)^{q} .
$$

Setting $v=u+1$, by direct calculation, we get

$$
\begin{aligned}
\int_{\Omega}\left(\Delta v^{\gamma}\right)^{2}= & \int_{\Omega} \gamma^{2} v^{2 \gamma-2}(\Delta v)^{2}+\int_{\Omega} \gamma^{2}(\gamma-1)^{2} v^{2 \gamma-4}|\nabla v|^{4} \\
& +2 \int_{\Omega} \gamma^{2}(\gamma-1) v^{2 \gamma-3} \Delta v|\nabla v|^{2}, \\
\int_{\Omega} \Delta v \Delta v^{q}= & \int_{\Omega} q(\Delta v)^{2} v^{q-1}+\int_{\Omega} q(q-1)|\nabla v|^{2} \Delta v v^{q-2} .
\end{aligned}
$$

From these two equalities and (3-4) we obtain

$$
\int_{\Omega}\left(\frac{q}{\gamma^{2}}\left(\Delta v^{\gamma}\right)^{2}-q(\gamma-1)^{2}|\nabla v|^{4} v^{2 \gamma-4}\right)+\int_{\partial \Omega} \frac{\partial(\Delta v)}{\partial n}=\int_{\Omega} \lambda v^{p+q} .
$$

For the second term in (3-5), we have 
(3-6)

$$
\begin{aligned}
\int_{\Omega}|\nabla v|^{4} v^{2 \gamma-4} & \\
& =\frac{1}{\gamma^{4}} \int_{\Omega} v^{-2 \gamma}\left|\nabla v^{\gamma}\right|^{4}=\frac{1}{\gamma^{4}} \int_{\Omega}\left|\nabla v^{\gamma}\right|^{2} \nabla v^{\gamma}\left(-\nabla v^{-\gamma}\right) \\
& =\frac{1}{\gamma^{4}} \int_{\Omega}\left(-\nabla \frac{\left|\nabla v^{\gamma}\right|^{2} \nabla v^{\gamma}}{v^{\gamma}}+\frac{\nabla\left(\left|\nabla v^{\gamma}\right|^{2}\right) \nabla v^{\gamma}}{v^{\gamma}}+\frac{\left|\nabla v^{\gamma}\right|^{2} \Delta v^{\gamma}}{v^{\gamma}}\right) \\
& =\frac{1}{\gamma^{4}} \int_{\Omega} v^{-\gamma} \nabla\left(\left|\nabla v^{\gamma}\right|^{2}\right) \nabla v^{\gamma}+\left|\nabla v^{\gamma}\right|^{2} \Delta v^{\gamma}-\frac{1}{\gamma} \int_{\partial \Omega} v^{2 \gamma-3}|\nabla v|^{2} \frac{\partial v}{\partial n} .
\end{aligned}
$$

A simple calculation yields

$$
\frac{1}{\gamma^{4}} \int_{\Omega} v^{-\gamma}\left|\nabla v^{\gamma}\right|^{2} \Delta v^{\gamma}=\frac{\gamma-1}{\gamma} \int_{\Omega} v^{2 \gamma-4}|\nabla v|^{4}+\frac{1}{\gamma} \int_{\Omega} v^{2 \gamma-3}|\nabla v|^{2} \Delta v .
$$

Substituting (3-7) into (3-6), we get

$$
\begin{aligned}
& \int_{\Omega}|\nabla v|^{4} v^{2 \gamma-4} \\
& \quad=\int_{\Omega} v^{2 \gamma-3}|\nabla v|^{2} \Delta v+\frac{1}{\gamma^{3}} \int_{\Omega} v^{-\gamma} \nabla\left(\left|\nabla v^{\gamma}\right|^{2}\right) \nabla v^{\gamma}-\int_{\partial \Omega}|\nabla v|^{2} \frac{\partial v}{\partial n} .
\end{aligned}
$$

We now estimate the second term on the right side of (3-8). From the proof of Lemma 2.3, together with the identity $\frac{1}{2} \Delta\left|\nabla v^{\gamma}\right|^{2}=\left|\nabla^{2} v^{\gamma}\right|^{2}+\left\langle\nabla \Delta v^{\gamma}, \nabla v^{\gamma}\right\rangle$, we have

$$
\begin{aligned}
\frac{1}{\gamma^{3}} \int_{\Omega} v^{-\gamma} \nabla\left(\left|\nabla v^{\gamma}\right|^{2}\right) \nabla v^{\gamma} \leq & \frac{1}{2} \int_{\Omega}|\nabla v|^{4} v^{2 \gamma-4}+\frac{2}{\gamma^{2}} \int_{\Omega}\left(\Delta v^{\gamma}\right)^{2} \\
& +\frac{1}{\gamma^{2}} \int_{\partial \Omega} \frac{\partial\left|\nabla v^{\gamma}\right|^{2}}{\partial n}-\frac{2}{\gamma^{2}} \int_{\partial \Omega}\left(\Delta v^{\gamma}\right) \frac{\partial v^{\gamma}}{\partial n} .
\end{aligned}
$$

By (3-8) and (3-9), thanks to the convexity of the domain $\Omega$, we get

$$
\begin{aligned}
& \frac{1}{2} \int_{\Omega}|\nabla v|^{4} v^{2 \gamma-4} \\
& \quad \leq \int_{\Omega} v^{2 \gamma-3}|\nabla v|^{2} \Delta v+\frac{2}{\gamma^{2}} \int_{\Omega}\left(\Delta v^{\gamma}\right)^{2}-(2 \gamma-1) \int_{\partial \Omega}|\nabla v|^{2} \frac{\partial v}{\partial n} .
\end{aligned}
$$

For the first term on the right side of (3-10), since $v=u+1$, we have $\Delta v=\Delta u<0$ by maximal principle, and the inequality

$$
\Delta v<-\sqrt{\frac{2 \lambda}{p+1}} v^{\frac{p+1}{2}}<0,
$$

by Lemma 3.2 of [Cowan et al. 2010]. Thus

$$
\int_{\Omega} v^{2 \gamma-3}|\nabla v|^{2} \Delta v \leq \int_{\Omega}-\sqrt{\frac{2 \lambda}{p+1}} v^{2 \gamma-3+\frac{p+1}{2}}|\nabla v|^{2} .
$$


Moreover, we have

$$
\begin{array}{r}
\int_{\Omega}-\sqrt{\frac{2 \lambda}{p+1}} v^{2 \gamma-3+\frac{p+1}{2}}|\nabla v|^{2}=-\int_{\Omega} \frac{\sqrt{\frac{2 \lambda}{p+1}}}{2 \gamma-2+\frac{p+1}{2}} \nabla\left(v^{2 \gamma-2+\frac{p+1}{2}} \nabla v\right) \\
+\int_{\Omega} \frac{\sqrt{\frac{2 \lambda}{p+1}}}{2 \gamma-2+\frac{p+1}{2}} v^{2 \gamma-2+\frac{p+1}{2}} \Delta v .
\end{array}
$$

For the second term on the right, using (3-11) again, we have

$$
\int_{\Omega} \frac{\sqrt{\frac{2 \lambda}{p+1}}}{2 \gamma-2+\frac{p+1}{2}} v^{2 \gamma-2+\frac{p+1}{2}} \Delta v \leq-\int_{\Omega} \frac{\frac{2 \lambda}{p+1}}{2 \gamma-2+\frac{p+1}{2}} v^{2 \gamma+p-1} .
$$

Hence, we obtain

$$
\int_{\Omega} v^{2 \gamma-3}|\nabla v|^{2} \Delta v \leq-\int_{\partial \Omega} \frac{\sqrt{\frac{2 \lambda}{p+1}}}{2 \gamma-2+\frac{p+1}{2}} \frac{\partial v}{\partial n}-\int_{\Omega} \frac{\frac{2 \lambda}{p+1}}{2 \gamma-2+\frac{p+1}{2}} v^{2 \gamma+p-1},
$$

where we used $\left.v\right|_{\partial \Omega}=u+\left.1\right|_{\partial \Omega}=1$, for the boundary term in (3-4), (3-10) and (3-12). By the remark after Theorem 3.1, we find that there exists a constant $C$ (the constant $C$ appeared now and later in this section is independent of $u$ ), such that

$$
\int_{\partial \Omega}\left(|\nabla u|^{2}\left|\frac{\partial u}{\partial n}\right|+\left|\frac{\partial(\Delta u)}{\partial n}\right|+\left|\frac{\partial u}{\partial n}\right|\right) \leq C .
$$

Combining (3-5), (3-10), (3-12) and (3-13), we get $\left(1-4(\gamma-1)^{2}\right) \int_{\Omega}\left(\Delta(u+1)^{\gamma}\right)^{2}+\left(\frac{8 \lambda \gamma^{2}(\gamma-1)^{2}}{(4 \gamma+p-3)(p+1)}-\frac{\lambda \gamma^{2}}{q}\right) \int_{\Omega}(u+1)^{p+q} \leq C$. If $1-4(\gamma-1)^{2}>0$ and

$$
p\left(1-4(\gamma-1)^{2}\right)+\frac{8 \gamma^{2}(\gamma-1)^{2}}{(4 \gamma+p-3)(p+1)}-\frac{\gamma^{2}}{q}>0
$$

and $u$ is a stable solution to (1-4), we have

$$
\left(p\left(1-4(\gamma-1)^{2}\right)+\frac{8 \gamma^{2}(\gamma-1)^{2}}{(4 \gamma+p-3)(p+1)}-\frac{\gamma^{2}}{2 \gamma-1}\right) \int_{\Omega}(u+1)^{p+q} \leq \frac{C}{\lambda} .
$$

This leads to $u+1 \in L^{p+q}$.

If $p+q>(p-1) n / 4$, then classical regularity theory implies that $u \in L^{\infty}(\Omega)$.

Therefore we have established the bound of extremal solutions of (1-4) if (3-14) is satisfied and

$$
p<\frac{8 \gamma+n-4}{n-4}
$$


By Lemma A.2 and Theorem 3.8 of [Wei and Ye 2010], we have proved that the extremal solution $u^{*}$, the unique solution of (1-4) (where $\lambda=\lambda^{*}$ ), is bounded provided that one of these conditions hold:

(1) If $n \leq 8$, then $p>1$.

(2) If $9 \leq n \leq 19$, there exists $\varepsilon_{n}>0$ such that for any $1<p<\frac{n}{n-8}+\varepsilon_{n}$.

(3) If $n \geq 20$, then $1<p<1+\frac{8 p^{*}}{n-4}$, where $p^{*}$ was defined immediately after Theorem 1.1.

\section{Appendix}

In this appendix, we study the inequalities

$$
p\left(1-4(\gamma-1)^{2}\right)-\frac{\gamma^{2}}{2 \gamma-1}+\frac{8 \gamma^{2}(\gamma-1)^{2}}{(4 \gamma-3+p)(p+1)}>0
$$

and

$$
p<\frac{8 \gamma+n-4}{n-4} .
$$

In order to get a better range for the power $p$ from (A-1) and (A-2), we must study the following equation obtained by letting $p=\frac{8 \gamma+n-4}{n-4}$ in (A-1):

$$
\frac{8 \gamma+n-4}{n-4}\left(1-4(\gamma-1)^{2}\right)-\frac{\gamma^{2}}{2 \gamma-1}+\frac{8 \gamma^{2}(\gamma-1)^{2}}{\left(4 \gamma-3+\frac{8 \gamma+n-4}{n-4}\right)\left(\frac{8 \gamma+n-4}{n-4}+1\right)}=0
$$

We need only consider the behavior of (A-3) for $\gamma \in\left(1, \frac{3}{2}\right)$. Through tedious computations, we see that the equation at the bottom of page 495 is the simplified form of (A-3). As a consequence, they have same roots in $\left(1, \frac{3}{2}\right)$.

We denote the left side of (A-3) by $h(\gamma)$. Notice that if $\gamma=\frac{n-4}{n-8}$, then $p=\frac{n}{n-8}$ and $\gamma-1=\frac{4}{n-8}$. Hence

$$
h\left(\frac{n-4}{n-8}\right)=\frac{8}{n-8}\left(n^{4}-18 n^{3}-56 n^{2}+384 n-512\right) .
$$

In fact, if $n=20$, then $h\left(\frac{4}{3}\right)=512>0$. On the other hand, it is also easy to see that $h\left(\frac{3}{2}\right)<0$, while it is obvious that $\left(4 \gamma-3+\frac{8 \gamma+n-4}{n-4}\right)\left(\frac{8 \gamma+n-4}{n-4}+1\right)>0$ and $(2 \gamma-1)>0$ when $\gamma \in\left(\frac{n-4}{n-8}, \frac{3}{2}\right)$. Therefore, by continuity, (A-3) possesses a root in $\left(\frac{n-4}{n-8}, \frac{3}{2}\right)$. We denote the smallest root of (A-3) greater than $\frac{n-4}{n-8}$ by $p^{*}$. Once we pick out a $\gamma$ from the interval $\left(\frac{n-4}{n-8}, p^{*}\right), h(\gamma)$ is of course positive. By continuity, we can find a small positive number $\delta$ such that the inequality

$$
p\left(1-4(\gamma-1)^{2}\right)-\frac{\gamma^{2}}{2 \gamma-1}+\frac{8 \gamma^{2}(\gamma-1)^{2}}{(4 \gamma-3+p)(p+1)}>0
$$


holds when $p \in\left(\frac{8 \gamma+n-4}{n-4}-\delta, \frac{8 \gamma+n-4}{n-4}\right)$. So, we conclude that when $\gamma$ runs in the whole interval $\left(\frac{n-4}{n-8}, p^{*}\right)$, the power $p$ can be in the whole interval $\left(\frac{n}{n-8}, 1+\frac{8 p^{*}}{n-4}\right)$. We summarize the result as follows:

Lemma A.2. When $n \geq 20$, the range of $p$ satisfying (A-1) and (A-2) equals $\left(\frac{n}{n-8}, 1+\frac{8 p^{*}}{n-4}\right)$, and this interval is not empty.

\section{References}

[Berchio and Gazzola 2005] E. Berchio and F. Gazzola, "Some remarks on biharmonic elliptic problems with positive, increasing and convex nonlinearities", Electron. J. Differential Equations 2005 (2005), Paper No. 34, 1-20. MR 2006e:35067 Zbl 1129.35349

[Cowan et al. 2010] C. Cowan, P. Esposito, and N. Ghoussoub, "Regularity of extremal solutions in fourth order nonlinear eigenvalue problems on general domains", Discrete Contin. Dyn. Syst. 28:3 (2010), 1033-1050. MR 2011e:35085 Zbl 1196.35152

[Farina 2007] A. Farina, "On the classification of solutions of the Lane-Emden equation on unbounded domains of $\mathbb{R}^{N}$,, J. Math. Pures Appl. (9) 87:5 (2007), 537-561. MR 2008c:35070 Zbl 1143.35041

[Ferrero et al. 2009] A. Ferrero, H.-C. Grunau, and P. Karageorgis, "Supercritical biharmonic equations with power-type nonlinearity", Ann. Mat. Pura Appl. (4) 188:1 (2009), 171-185. MR 2010g: 35087 Zbl 1179.35125

[Gazzola and Grunau 2006] F. Gazzola and H.-C. Grunau, "Radial entire solutions for supercritical biharmonic equations”, Math. Ann. 334:4 (2006), 905-936. MR 2007b:35114 Zbl 1152.35034

[Gui et al. 1992] C. Gui, W.-M. Ni, and X. Wang, "On the stability and instability of positive steady states of a semilinear heat equation in $\mathbb{R}^{n} "$, Comm. Pure Appl. Math. 45:9 (1992), 1153-1181. MR 93h:35095 Zbl 0811.35048

[Guo and Webb 2002] Z. Guo and J. R. L. Webb, "Large and small solutions of a class of quasilinear elliptic eigenvalue problems”, J. Differential Equations 180:1 (2002), 1-50. MR 2002k:35246 Zbl 1014.35030

[Guo and Wei 2009] Z. Guo and J. Wei, "On a fourth order nonlinear elliptic equation with negative exponent”, SIAM J. Math. Anal. 40:5 (2009), 2034-2054. MR 2010b:35125 Zbl 1175.35144

[Guo and Wei 2010] Z. Guo and J. Wei, "Qualitative properties of entire radial solutions for a biharmonic equation with supercritical nonlinearity", Proc. Amer. Math. Soc. 138:11 (2010), 39573964. MR 2012a:35054 Zbl 1203.35105

[Lin 1998] C.-S. Lin, "A classification of solutions of a conformally invariant fourth order equation

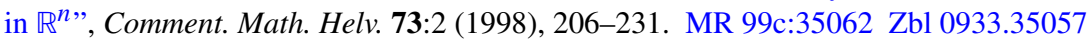

[Troy 1981] W. C. Troy, "Symmetry properties in systems of semilinear elliptic equations", $J$. Differential Equations 42:3 (1981), 400-413. MR 83b:35051 Zbl 0486.35032

[Wei 1996] J. Wei, “Asymptotic behavior of a nonlinear fourth order eigenvalue problem”, Comm. Partial Differential Equations 21:9-10 (1996), 1451-1467. MR 97h:35066 Zbl 0872.35013

[Wei and Xu 1999] J. Wei and X. Xu, "Classification of solutions of higher order conformally invariant equations", Math. Ann. 313:2 (1999), 207-228. MR 2000a:58093 Zbl 0940.35082

[Wei and Ye 2010] J. Wei and D. Ye, "Liouville theorems for finite Morse index solutions of biharmonic problem", preprint, 2010, http://www.math.cuhk.edu.hk/ wei/4thstable29-10-10.pdf. To appear in Math. Ann. 
[Xu 2000] X. Xu, "Uniqueness theorem for the entire positive solutions of biharmonic equations in $\mathbb{R}^{n}$ ", Proc. Roy. Soc. Edinburgh Sect. A 130:3 (2000), 651-670. MR 2001f:35143 Zbl 0961.35037

Received November 1, 2011. Revised April 24, 2012.

JUNCHENG WEI

DEPARTMENT OF MATHEMATICS

The Chinese University of Hong Kong

SHATIN, NT

HONG KONG

wei@math.cuhk.edu.hk

Xingwang Xu

DEPARTMENT OF MATHEMATICS

NATIONAL UNIVERSITY OF SINGAPORE

BLOCK S17 (SOC1)

10 LOWER KENT RIDGE ROAD

SINGAPORE 119076

SINGAPORE

matxuxw@nus.edu.sg

WEN YANG

DEPARTMENT OF MATHEMTICS

Chinese University of Hong Kong

SHATIN, NT

HONG KONG

wyang@math.cuhk.edu.hk 


\title{
PACIFIC JOURNAL OF MATHEMATICS
}

\author{
msp.org/pjm
}

Founded in 1951 by E. F. Beckenbach (1906-1982) and F. Wolf (1904-1989)

\section{EDITORS}

V. S. Varadarajan (Managing Editor)

Department of Mathematics

University of California

Los Angeles, CA 90095-1555

pacific@math.ucla.edu

Paul Balmer

Department of Mathematics

University of California

Los Angeles, CA 90095-1555

balmer@math.ucla.edu

Daryl Cooper

Department of Mathematics

University of California

Santa Barbara, CA 93106-3080 cooper@math.ucsb.edu

Jiang-Hua $\mathrm{Lu}$

Department of Mathematics

The University of Hong Kong

Pokfulam Rd., Hong Kong jhlu@maths.hku.hk
Don Blasius

Department of Mathematics University of California

Los Angeles, CA 90095-1555

blasius@math.ucla.edu

Robert Finn

Department of Mathematics Stanford University

Stanford, CA 94305-2125

finn@math.stanford.edu

Sorin Popa

Department of Mathematics

University of California

Los Angeles, CA 90095-1555

popa@math.ucla.edu

Paul Yang

Department of Mathematics

Princeton University

Princeton NJ 08544-1000

yang@math.princeton.edu

\section{PRODUCTION}

Silvio Levy, Scientific Editor, production@msp.org

\section{SUPPORTING INSTITUTIONS}

ACADEMIA SINICA, TAIPEI

CALIFORNIA INST. OF TECHNOLOGY

INST. DE MATEMÁTICA PURA E APLICADA

KEIO UNIVERSITY

MATH. SCIENCES RESEARCH INSTITUTE

NEW MEXICO STATE UNIV.

OREGON STATE UNIV.

\author{
STANFORD UNIVERSITY \\ UNIV. OF BRITISH COLUMBIA \\ UNIV. OF CALIFORNIA, BERKELEY \\ UNIV. OF CALIFORNIA, DAVIS \\ UNIV. OF CALIFORNIA, LOS ANGELES \\ UNIV. OF CALIFORNIA, RIVERSIDE \\ UNIV. OF CALIFORNIA, SAN DIEGO \\ UNIV. OF CALIF., SANTA BARBARA
}

\author{
Vyjayanthi Chari \\ Department of Mathematics \\ University of California \\ Riverside, CA 92521-0135 \\ chari@math.ucr.edu \\ Kefeng Liu \\ Department of Mathematics \\ University of California \\ Los Angeles, CA 90095-1555 \\ liu@math.ucla.edu \\ Jie Qing \\ Department of Mathematics \\ University of California \\ Santa Cruz, CA 95064 \\ qing@cats.ucsc.edu
}

These supporting institutions contribute to the cost of publication of this Journal, but they are not owners or publishers and have no responsibility for its contents or policies.

See inside back cover or msp.org/pjm for submission instructions.

The subscription price for 2013 is US \$400/year for the electronic version, and \$485/year for print and electronic.

Subscriptions, requests for back issues and changes of subscribers address should be sent to Pacific Journal of Mathematics, P.O. Box 4163, Berkeley, CA 94704-0163, U.S.A. The Pacific Journal of Mathematics is indexed by Mathematical Reviews, Zentralblatt MATH, PASCAL CNRS Index, Referativnyi Zhurnal, Current Mathematical Publications and the Science Citation Index.

The Pacific Journal of Mathematics (ISSN 0030-8730) at the University of California, c/o Department of Mathematics, 798 Evans Hall \#3840, Berkeley, CA 94720-3840, is published monthly except July and August. Periodical rate postage paid at Berkeley, CA 94704, and additional mailing offices. POSTMASTER: send address changes to Pacific Journal of Mathematics, P.O. Box 4163, Berkeley, CA 94704-0163.

PJM peer review and production are managed by EditFLOW ${ }^{\circledR}$ from Mathematical Sciences Publishers.

PUBLISHED BY

mathematical sciences publishers

nonprofit scientific publishing

http://msp.org/

(C) 2013 Mathematical Sciences Publishers 


\section{PACIFIC JOURNAL OF MATHEMATICS}

Volume $263 \quad$ No. $2 \quad$ June 2013

Realizations of $B C_{r}$-graded intersection matrix algebras with grading 257 subalgebras of type $B_{r}, r \geq 3$

SANDEEP BHARGAVA and YUN GAO

Stable flags, trivializations and regular connections

283

Elie COMPOINT and EDUARDo COREL

Elliptic aliquot cycles of fixed length

353

NATHAN JONES

Asymptotic $L^{4}$ norm of polynomials derived from characters

DANIEL J. KATZ

Degree-three spin Hurwitz numbers

JUNHO LEE

$\left(\mathbb{Z}_{2}\right)^{3}$-colorings and right-angled hyperbolic 3-manifolds

YOULIN LI and JiMING MA

Real closed separation theorems and applications to group algebras

Tim NETZER and ANDREAS THOM

Uniqueness theorem for ordinary differential equations with Hölder continuity

YifEI PAN, MEI WANG and Yu YAN

An analogue to the Witt identity

G. A. T. F. DA CostA and G. A. ZimmermanN

On the classification of stable solutions to biharmonic problems in large dimensions

Juncheng Wei, Xingwang Xu and Wen Yang 